\title{
Gestión ambiental aplicada al planeamiento de proyectos mineros
}

\author{
Max Schwarz \\ B\&G Engineering S.A.C. \\ Ingeniería Industrial n. ${ }^{\circ}$ 29, 2011, ISSN 1025-9929, pp. 99-123 \\ Recibido: 8 de febrero del 2011 / Aprobado: 19 de abril del 2011
}

\begin{abstract}
Resumen: El artículo muestra parte de la experiencia lograda en el Perú con la aplicación de herramientas de gestión ambiental al sector minero local, enfocándose en la incorporación de variables ambientales al proceso de planeamiento minero con la finalidad de optimizar, desde el diseño, el logro de un proceso eficiente con un mejor consumo de recursos y con la consecuente emisión controlada de residuos al medioambiente. Se hace una descripción de la importancia del uso de herramientas de gestión ambiental a lo largo del ciclo de vida de las operaciones mineras, desarrollando el impacto sobre la inversión (CAPEX) y el costo de operación (OPEX), para el modelo de negocio extractivo en el Perú.
\end{abstract}

Palabras clave: minería / gestión ambiental / mejora continua / costos / seguridad / medioambiente / planificación / Perú

\section{Environmental management applied to mining projects planning}

Abstract: The article shows about the Peruvian experience with the implementation of environmental management tools to the local mining sector by focusing on the incorporation of environmental variables to the mine planning process in order to optimize an efficient design process with a better use of resources and the consequent waste controlled into the environment. A description of using the environmental management tools into the life cycle of mining operations and the influence on capital cost investment (CAPEX) and operating cost (OPEX) for the business model extraction in Peru.

Keywords: mining / environmental management / continuous improvement / costos / security / environment / planning / Peru 


\section{INTRODUCCIÓN}

Desde 1990 la minería peruana formal se ha visto positivamente afectada por la aparición de variables adicionales a las operaciones y técnicas tradicionales, de manera que hoy la variable ambiental, la seguridad industrial y la variable social (o sociocomunal actual) condicionan seriamente la existencia del negocio y se constituyen en tema central y estratégico que debe superarse necesariamente antes de operar algún proyecto minero.

A inicios de 1990 surgen las primeras regulaciones ambientales para la minería en el Perú, emulando, de alguna manera, las regulaciones estadounidenses, canadienses y sudafricanas en materia de seguridad, salud y medioambiente, para luego reglamentar el agua, el aire, la generación eléctrica, los vertimientos y la elaboración de proyectos nuevos. En 1993 surge el primer Reglamento de Protección Ambiental de las Actividades Minero-Metalúrgicas (DS016-93-EM), que planteaba que, a partir de esa fecha, las unidades mineras en operación deberían completar un PAMA (Programa de Adecuación al Medioambiente) para adecuarse a la nueva legislación; en cambio, las unidades que se creaban a partir de esa fecha deberían nacer directamente con un Estudio de Impacto Ambiental (EIA), en el cual pueda planearse de manera cuidadosa que estén adecuadas, técnicamente, desde su diseño, a la nueva reglamentación ambiental.

En 1996, y tomando como base las normas británicas de gestión ambiental (BS7750), el Comité Técnico 207 de la Organización Internacional de Estándares (ISO) generó las primeras normas ISO aplicadas a la gestión ambiental (Serie ISO14000), de las cuales la única norma certificable era el ISO14001:96. A partir de ahí numerosas organizaciones alrededor del mundo avanzaron en diseñar, implementar y mantener sistemas de gestión ambiental bajo este estándar internacional. Años más tarde, la norma ISO14001:96 fue mejorada y cambiada de versión, evolucionando en función de la experiencia ganada en la implementación del ISO14001:2004, que en la actualidad opera formalmente como norma certificable vigente.

En materia de seguridad industrial aplicada a la minería, también ha habido una evolución debida fundamentalmente a acciones correctivas tomadas como consecuencia de la enorme cantidad de accidentes 
fatales que la minería subterránea y de superficie provocan anualmente. A finales del 2001 se aprobó el Reglamento de Seguridad e Higiene Minera (DS046-2001-EM) para regular las actividades ligadas al riesgo, y generar la estructura estándar de las organizaciones de seguridad, estableciéndose especificaciones que deberían conducir a administrar niveles de riesgo razonables.

En 1999 se intentó mundialmente avanzar hacia Sistemas de Gestión de Riesgo tipo ISO para Seguridad, sin embargo, el proceso era cada vez más complejo y existían intereses creados por la existencia previa de sistemas de seguridad, instituidos fundamentalmente para la minería (NOSA e ISTEC) en países como Sudáfrica, que no permitieron la evolución de un Sistema de Gestión de Riesgos tipo ISO. No obstante, la presión por tener estándares internacionales asociados a la seguridad y la salud permitieron el surgimiento de la norma $\mathrm{OH}$ SAS18001:99 (Sistema de Gestión de Riesgos de Seguridad y Salud) como complemento para abarcar los temas de Seguridad Industrial en las organizaciones, hoy esta versión ha evolucionado hasta $\mathrm{OH}$ SAS18001:2007.

En la actualidad se encuentra bastante difundida la aplicación de sistemas individuales de gestión sea ambientales o de seguridad industrial, por lo que muchas compañías ya cuentan con uno funcionando o están en proceso de implementación para operar uno en el futuro. Con ello se ha iniciado a la vez una ola creciente de integraciones de los sistemas individuales de gestión en la búsqueda de mejoras significativas y mayor eficiencia para las organizaciones.

El diseño e implementación de sistemas integrados de gestión ambiental y seguridad industrial es hoy muy importante para la viabilidad de los proyectos mineros, dada la necesidad de tener organizada, controlada y evidenciada ante terceras partes interesadas la gestión sobre la seguridad industrial humana y el medioambiente en el que opera la organización.

Para ello, es necesario contar con sistemas de gestión que permitan obtener niveles de riesgo razonables, que se requieren como prácticas mundialmente aceptables. Por otro lado, es necesario conocer y comprender la naturaleza íntima de los sistemas de gestión para poder mejorarlos, encontrando una rentabilidad apropiada como producto de su implementación en el ciclo de vida del negocio. 


\section{PLANEAMIENTO AMBIENTAL: ¿POR QUÉ PLANEAR?}

La importancia de los procesos de planificación para el medioambiente nos hacen centrar la mirada en el concepto de prevención ambiental antes que en el de control ambiental, que encierra más bien conceptos puramente correctivos. El diseño de productos y procesos ofrece siempre importantes oportunidades de mejora con la introducción de variables ambientales al diseño de los proyectos, que permiten minimizar la emisión de los futuros residuos y planear la eficiente utilización de los recursos, reduciendo así los posibles impactos ambientales que pudieran generarse como consecuencia del desarrollo del proyecto o de las actividades de la empresa.

Por ello, la planificación se vuelve crítica para el medioambiente. Consideremos por un momento que un superintendente de mina organiza deficientemente su plan de minado; ello traerá como consecuencia inmediata una mayor utilización de maquinaria, consumo de combustibles, horas-hombre, repuestos y servicios conexos no necesariamente relevantes. Esto significa un uso ineficiente de los recursos y, como consecuencia, una mayor generación de residuos, que en realidad es una falta de compromiso con el medioambiente. De igual forma puede suceder con las áreas de Ingeniería, Mantenimiento, Planta, Administración, Seguridad y Medioambiente al no contemplar la importancia crítica que tiene la planificación para cualquier proyecto.

\section{LA IMPORTANCIA DEL DISEÑO}

Desde el punto de vista conceptual, el diseño de nuevos proyectos mineros involucra la consideración de criterios tecnológicos, económicofinancieros, legales, políticos, comerciales, logísticos, ambientales y de seguridad. Estos criterios permitirán definir la viabilidad del proyecto en el corto, mediano y largo plazos, sentando las bases de la futura organización. Cada uno de ellos incluirá variables ambientales, lo cual implica la consideración mínima de los siguientes elementos:

- Diseño tecnológico con variables ambientales (circuito cerrado, optimización de flujos, recirculación, reciclaje, reutilización, etcétera) donde sea posible aplicarlas.

- Utilización de ecomateriales o ecoproductos (degradables, biodegradables, fotodegradables, ecológicos, de tecnología combinada, reciclables, reutilizables, recargables, conservativos, ahorrativos, 
etcétera) donde sea posible, viable y adecuado en armonía con el medioambiente.

- Uso eficiente de los recursos (materias primas, insumos, recursos naturales, aire, agua, suelo, fuentes de energía y recursos humanos).

- Disposición y administración efectiva de residuos (sólidos, líquidos, gaseosos, calor, ruido y vibraciones). Ello incluye el planeamiento para el Almacén de Residuos, que permita recibir, registrar, clasificar y disponer (reciclar, reutilizar, vender o confinar) los diferentes residuos que se han de generar en el proyecto.

- Diseño de control del producto no conforme en todas las etapas del ciclo de vida.

- Diseño de control operacional de las actividades, procesos, productos y servicios de mayor relevancia y sobre los que se espera se puede tener influencia.

La Ingeniería Básica y la Ingeniería de Detalle deberán incorporar, donde sea posible, el concepto del circuito cerrado y el principio de la máxima reutilización. Estos conceptos deben considerarse desde el diseño, con el fin de garantizar la mínima salida hacia el medioambiente. Ello involucra la consideración de los siguientes criterios:

- Aislamiento del suelo con geomembranas, tanto para las pozas de solución como para las instalaciones minerometalúrgicas, pads y plantas de servicios, así como drenajes, alcantarillas y líneas de distribución o transporte de soluciones.

- Diseño de flujos líquidos y gaseosos, garantizando las mínimas posibilidades de salida hacia el medioambiente.

- Reutilización de flujos para garantizar una optimización en el enriquecimiento de las soluciones y asegurar donde sea posible una adecuada eficiencia energética y de recursos.

- Sistemas de instrumentación y control para el medioambiente y las variables operacionales asociadas con acceso remoto y habilitación para condiciones anormales y de emergencia que pudieran presentarse.

- Encapsulamiento final de materiales peligrosos con el diseño de eficientes botaderos de desmonte o material estéril que puedan generarse como producto de las actividades mineras, y la correcta disposición de los residuos sólidos químicos y no químicos. Ello debe sen- 
tar las bases para una futura revegetación de botaderos de acuerdo con el Plan de Cierre que pueda trazarse por la organización.

\section{PLANEAMIENTO DE LA ORGANIZACIÓN}

Las empresas mineras son asociaciones con fines mercantiles enfocadas en actividades de extracción, beneficio, producción y comercialización de recursos minerales (recursos primarios no renovables) del suelo y el subsuelo de la corteza terrestre. La minería es una actividad extractiva clasificada como primaria por su naturaleza (generalmente es considerada así al tratarse de una actividad que no brinda mayor valor agregado que la extracción y beneficio mismo del mineral para obtener metales en forma de concentrados o como metal en barra, pero que, sin embargo, por la envergadura del negocio constituye la principal fuente generadora de ingresos por exportaciones y divisas del Perú). Por su naturaleza general (especialmente las minerías grande y mediana) tiende a tener las siguientes características relevantes:

- Es una industria altamente mecanizada con uso intensivo y creciente de tecnología.

- Hace uso intensivo de agua, materiales, energía y productos e insumos industriales.

- Baja intensidad en mano de obra.

- Recurso humano altamente especializado y calificado.

- Alto potencial de impacto socioambiental en los lugares donde opera.

- Es una industria de alto riesgo que requiere gran especialización y concentración.

- Es una industria altamente sensible a los precios internacionales de los metales; es decir, maneja el costo pero no el precio.

En términos generales, la actividad minera es muy rentable cuando se acerca a optimizar las características descritas anteriormente de la forma de operar de las minas grandes y medianas, pero teniendo especial consideración en la etapa del ciclo de vida en el que se encuentre la actividad minera en particular. Estas etapas requieren ser cuidadosamente estudiadas para ser incorporadas al análisis económico, social, cultural y ambiental de la actividad. Por ello, para entender este proceso es necesario comprender previamente las distintas etapas del ciclo 
de vida de las minas, donde claramente puede diferenciarse, como se observa en el gráfico 1, las siguientes etapas secuenciales:

- Preplaneación

- Diseño/modelación/factibilidad

- Construcción

- Prueba/ajuste/arranque

- Operación

- Cierre

- Poscierre



Elaboración propia. 
Existe una etapa inicial que es la fuente generadora de proyectos denominada "fase exploratoria" o "exploraciones" y que es el conjunto de actividades geológicas que definen e indican la existencia de recurso mineralizado con características comerciales. Esta etapa, que es la base de la minería, es el motor que repone las reservas agotables y sale a la búsqueda de nuevas oportunidades y nuevos proyectos geológicos económicamente interesantes para las organizaciones mineras. Las etapas de exploración generativa de recursos y reservas permiten determinar los inventarios reales de mineral que han de constituir la base de la reserva y el futuro de las operaciones mineras.

Luego de confirmada la fase exploratoria se inicia el proceso de preplaneación, el cual constituye el primer proceso operativo a partir de un proyecto geológico con características atractivas para su desarrollo. En esta etapa se organiza la confirmación de la existencia de reservas y se evalúan los elementos de riesgo que podrían afectar el futuro del proyecto minero, determinándose las principales responsabilidades globales del manejo futuro del proyecto (designación del responsable del proyecto) para su etapa de planificación y diseño.

La planificación, diseño o etapa de planeación es aquella en la cual se examina la factibilidad del proyecto; para ello, es necesario revisar con detalle la documentación legal y regulatoria respecto de la propiedad; los permisos ambientales y sociales locales, regionales, nacionales, internacionales y corporativos; y la determinación técnica de la factibilidad económica, tecnológica, social y ambiental del proyecto. Esta etapa es decisiva para avanzar en un proyecto determinado o detenerlo hasta alcanzar mejores resultados de la investigación geológica, o bien descartarlo por sus propias características, si fuera el caso. Se deben realizar todos los estudios técnicos y de factibilidad o regulatorios que sean aplicables para tomar la decisión sobre si poner o no en funcionamiento un proyecto determinado. Esta constituye, además, una etapa decisiva para determinar si se realiza o no el proyecto, pues el Banco y las entidades financieras fijan las inversiones a ejecutarse en función de los resultados de la factibilidad del proyecto minero y sobre la base de las reservas probadas-probables que puedan establecerse con suficiencia técnica por los especialistas.

La etapa de construcción es muy especial, pues involucra la presencia intensiva de operadores y contratistas que se convocan únicamente para la ejecución y supervisión de obras civiles, metalmecánicas, eléctricas e instrumentación y control (I\&C) que sean necesarios para 
poner a operar el proyecto. Este proceso incluye los pasos que se requieren para determinar, presentar y ejecutar (o controlar, dependiendo del rol desempeñado) el movimiento de tierras, la preparación de bases y estructuras, la construcción de edificios, el montaje, instalación y puesta en marcha de equipos y controles principales, de servicios y complementarios o auxiliares que permitan la operatividad del proyecto en condiciones normales, anormales y de emergencia, incluyendo las pruebas necesarias para el arranque.

La etapa siguiente es la operación minera propiamente dicha, donde se dan las operaciones unitarias elementales de la ingeniería de minas: perforación, voladura, carguío, transporte, beneficio y acondicionamiento de metales para su comercialización final, así como operaciones unitarias de servicios principales y de apoyo (bombeo, ventilación, etcétera) y cuya duración en el tiempo depende exclusivamente de las reservas reales que la mina pueda ofrecer hasta su agotamiento económico natural.

Cuando las reservas de una mina se agotan se debe proceder al inicio del cierre de la mina. El proceso de cierre de minas es bastante complejo; involucra alta tecnología y experiencia para su desarrollo. Se busca fundamentalmente dotar de estabilidad física y geoquímica para el largo plazo de todos los recursos y emplazamientos utilizados, así como el retorno a las condiciones preoperacionales (o lo más cercano a ello) de todas las habilitaciones consideradas desde su diseño. Este proceso incluye los de cierre social y legal regulatorio ante las autoridades de aplicación.

Finalmente, está la etapa del monitoreo poscierre, necesario para asegurar la estabilidad de las variables en el largo plazo, lo cual implica un proceso planeado y documentado, que requiere ser aprobado regulatoriamente para el seguimiento de variables ambientales y programas de monitoreo que evalúen la efectividad del cierre de mina previamente ejecutado.

El proceso minero tradicional implica el desarrollo de las operaciones unitarias propias de la ingeniería de minas, y se definen por la perforación, la voladura, el carguío, el transporte, el beneficio y la comercialización, tal como se muestra en la siguiente figura, correspondiente a un esquema convencional: 
Figura 2

Proceso productivo del ciclo de minado

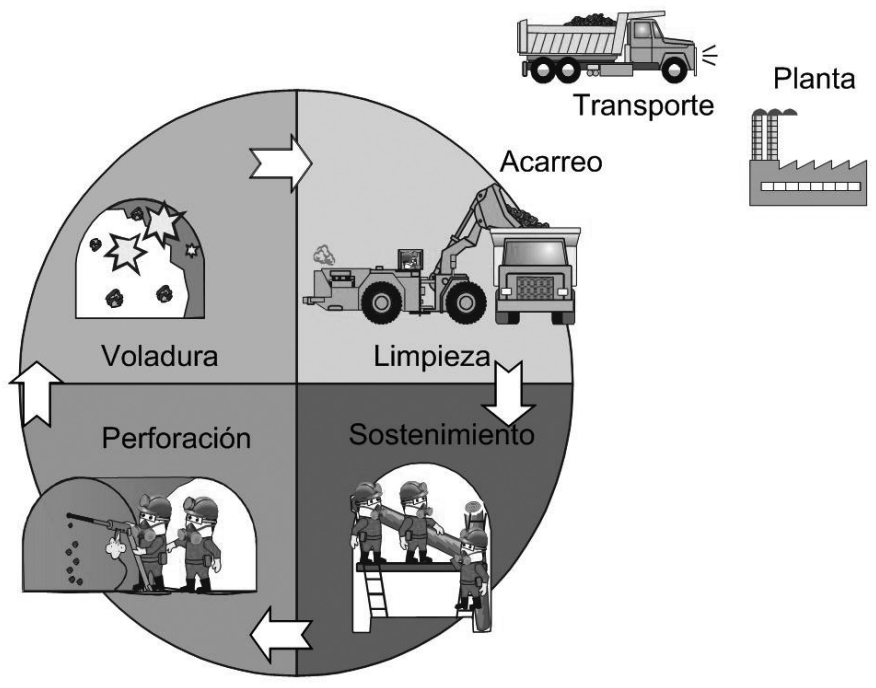

Elaboración propia.

\section{SISTEMAS DE GESTIÓN AMBIENTAL: ¿CÓMO NOS ORGANIZAMOS?}

El diseño nunca está completo si no contempla la variable organización. Ello implica la necesidad de generar una adecuada plataforma de organización. En este marco la introducción de un sistema de gestión ambiental (SGA) se vuelve prioritaria para garantizar un adecuado manejo de los diferentes aspectos ambientales presentes, pasados (pasivo ambiental) y futuros (nuevas instalaciones) en condiciones normales, anormales y de emergencia, tanto para las actividades, procesos y servicios como para los recursos, productos y residuos que la organización pueda generar y sobre los que se espera tener algún grado de influencia. Actualmente, en el Perú las experiencias de implementación del SGA han dejado interesantes contribuciones locales de gestión en beneficio del medioambiente y de la organización en su conjunto.

\section{SGA-ISO14001: HACIA UNA GESTIÓN DE CLASE MUNDIAL}

La introducción de SGA basados en el ISO14001 en las organizaciones mineras permite concentrar los esfuerzos organizacionales hacia 
Figura 3

SGA ISO14001: Modelo de gestión

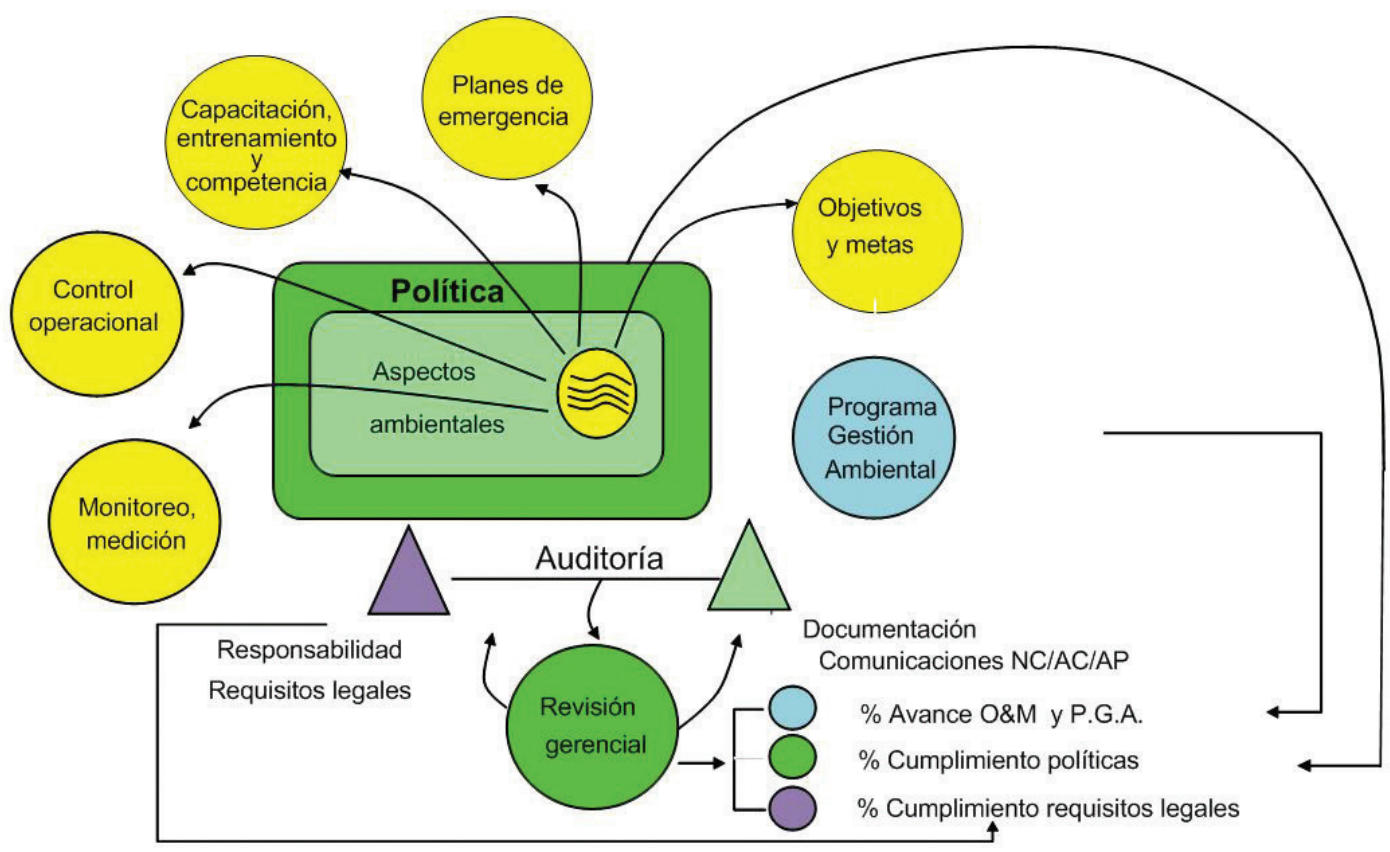

Elaboración propia.

los aspectos ambientales de mayor importancia en un sistema modular que incorpora la mejora continua en cada una de sus etapas y ciclos (véase la figura 3). Es así como pueden trazarse objetivos claros y metas ambientales precisas sobre las cuales orientar los esfuerzos de gestión. En realidad no se trata de un estudio ni de un software, sino más bien de una forma de trabajar introduciendo una cultura de calidad ambiental entre el personal y desarrollando un esquema claro que permita llevar a cabo en secuencia las actividades de planear, medir, controlar, revisar y mejorar en un ciclo continuo las actividades críticas sobre las que se pueda tener influencia.

Quizá lo más interesante se centre en la posibilidad de trazar objetivos y metas ambientales que puedan enfocar los principales compromisos que deberían consignarse en una política ambiental basada en el ISO14001. Estos compromisos podrían resumirse de la siguiente forma: 
- Compromiso con el medioambiente.

- Compromiso con la ley y la regulación asociada vigente.

- Compromiso con la mejora continua.

- Compromiso con la prevención de la contaminación y el uso eficiente de recursos.

- Compromiso con la formación, capacitación y entrenamiento.

Por ello, los objetivos y las metas ambientales deben estar en concordancia con los compromisos de la política ambiental que la organización se plantea. A continuación mostramos algunos ejemplos de cómo debería considerarse ello:

- Ejemplo 1: Objetivos referidos al uso eficiente de los recursos y prevención de la contaminación ambiental.

Objetivo: Optimizar el consumo de energía eléctrica/combustibles.

Meta: De $25 \mathrm{kw}-\mathrm{h} / \mathrm{t}$ a $20 \mathrm{kw}-\mathrm{h} / \mathrm{t}$

Meta: De 3,07 gal/onza a 2,98 gal/onza

(Nota: Obsérvese que la meta no busca consumir menos, sino consumir mejor con la consecuente optimización de los ratios en el largo plazo que la propia organización plantee para su Programa de Gestión Ambiental. De igual forma, obsérvese que la metodología busca asignar el consumo de los recursos a cada unidad de producto de la organización).

- Ejemplo 2: Objetivos referidos al cumplimiento de requisitos legales y regulaciones asociadas al medioambiente.

Objetivo: Cumplir siempre con el límite máximo permisible (LMP) en la descarga líquida de la solución de concentración de plomo al medioambiente, de acuerdo con la regulación peruana (RM 011-96 $\mathrm{EM} / \mathrm{VMM})$.

Meta: El 100\% de las mediciones con concentración de plomo menor a $\operatorname{LMP}(0,4 \mathrm{mg} / \mathrm{l})$.

- Ejemplo 3: Objetivos referidos a la formación, capacitación y entrenamiento.

Objetivo: Capacitación del 100\% del personal en gestión ambiental. Meta: Mínimo 5 h-h/mes al 100\% del personal. 
- Ejemplo 4: Objetivos referidos a la mejora continua.

Objetivo: Ejecutar eficientemente las acciones correctivas tomadas.

Meta: Mínimo 90\% de las acciones correctivas tomadas por mes con 95\% de validez de eficacia en el reporte mensual.

Esta forma de trazar objetivos y metas logra incorporar a toda la organización en un verdadero trabajo de equipo con objetivos individuales y objetivos compartidos entre las diferentes áreas de la organización, con el consecuente balance de los esfuerzos entre todos los involucrados. De igual forma, permite obtener interesantes beneficios económicos determinados por los importantes ahorros que se generan por la utilización eficiente de los recursos y la gestión efectiva de los residuos. Estos ahorros son fácilmente cuantificables con un retorno de corto plazo e interesantes rentabilidades económico-financieras.

Los procesos de mejora continua involucran las actividades secuenciales básicas de planear, medir, controlar, revisar y mejorar. Ello significa recordar dos grandes frases de la cultura de gestión de clase mundial: "Lo que no se planifica no puede administrarse..." y "Lo que no se mide no se controla y lo que no se controla no puede administrarse...”. Este proceso conlleva la necesidad de aprender de los errores y considerar las adversidades como interesantes oportunidades de mejora. Ello pasa necesariamente por la etapa de la revisión que permite identificar las oportunidades de mejora en el corto, mediano y largo plazos para la organización.

En los sistemas de gestión ambiental basados en el ISO14001 existen grandes elementos de mejora continua que se han venido introduciendo. Algunos ejemplos de ello son:

- La plataforma documentaria que por medio de solicitudes de cambio y codificación de documentos permite evidenciar las nuevas versiones en la documentación de los sistemas.

- Las acciones correctivas y preventivas que permiten evidenciar los cambios reales ante la detección de no conformidades reales o potenciales, tratando no solo de levantar la no conformidad, sino, fundamentalmente, buscando eliminar la raíz de la causa de esta por medio de la periódica validez de eficacia de dichas acciones. 
Sin embargo, la mejora continua es aún más completa al convertirse en un verdadero mecanismo de perfeccionamiento permanente que se alimenta de la experiencia de cada día en el uso del sistema y que permite la participación directa del usuario de cada procedimiento o instrucción de trabajo en su continuo rediseño y mejora. En realidad, los problemas y las adversidades presentan en sí mismos importantes oportunidades de mejora que el sistema enseña a reconocer para beneficio de la organización.

\section{ENFOQUES DE COSTO-GESTIÓN DE LA EFECTIVIDAD}

\subsection{Costos de seguridad y medioambiente}

\subsubsection{Ingreso ambiental}

En cuanto a los ingresos, es necesario entender que las oportunidades de rentabilidad ambiental y de seguridad (en términos de riesgo) están previstas para capitalizar ahorros importantes en lo que se refiere al uso de agua, energía, materiales y métodos de operación, con la finalidad de optimizar los estándares trazados hacia el producto final, reducir los tiempos de los procesos y asegurar la administración de un nivel de riesgo razonable. Para ello debe entenderse que las metas ambientales y de seguridad son metas globales e involucran a toda la organización en su conjunto y no solamente a las áreas técnicas tradicionales de producción, operaciones, procesos o planta.

En esencia, la asociación, en términos de indicadores de gestión o ratios de las relaciones recurso/producto y residuo/producto, puede ser perfectamente optimizada y es susceptible de gestión y mejora con la implementación de programas de gestión de riesgo (PGR) de seguridad y medioambiente. En ese sentido, la optimización de variables de recursos y residuos asociados al producto final, que la mina puede ofrecer en términos de $\mathrm{kw}-\mathrm{h} / \mathrm{t}, \mathrm{m}^{3} / \mathrm{t}, \mathrm{KgQx} / \mathrm{t}$, entre otros muchos susceptibles de optimización, pueden generar una ventaja competitiva en el sector.

Lo importante es que en este contexto los pequeños ahorros en la optimización de los consumos permiten obtener grandes ahorros globales de dinero en la organización que justifican rentablemente todos los cambios y mejoras implementadas con una sólida rentabilidad ambiental, de seguridad-riesgo y a la vez económica-financiera en términos de ahorro real. 
Se puede describir la estructura de ingresos basada en la interpretación de ingreso ambiental definida en el alcance de esta investigación en la figura 4:

\section{Figura 4}

Ingresos ambientales

1. Ingresos por el uso eficiente de recursos
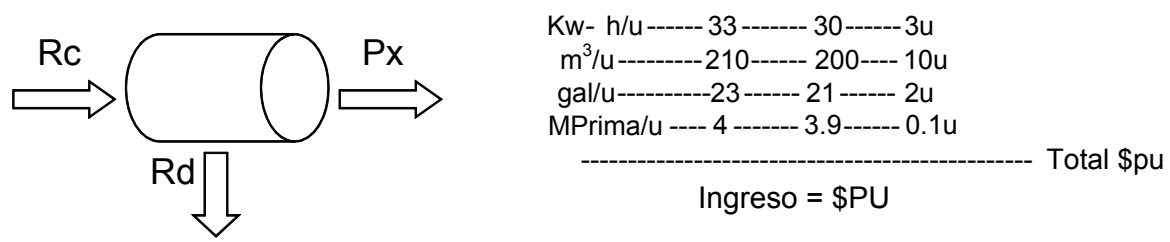

2. Ingresos ambientales por rentas pasivas

- Bonos de carbono (5 - 7 \$/tC): Forestal, combustibles, emisiones, suelo

- Usos turísticos en renta pasiva: Alquileres, rentas, impacto visual, etc.

3. Ingresos por la gestión efectiva de residuos

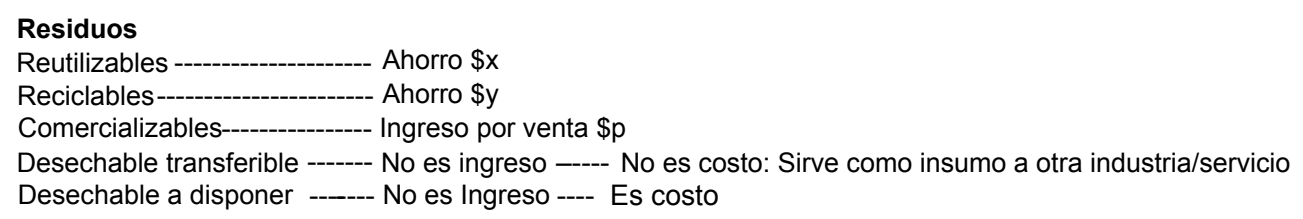

Elaboración propia.

La aplicación de técnicas para contabilizar ahorros importantes en la gestión ambiental empresarial genera un fondo de caja suficiente para enfrentar los costos ambientales que se presenten y determinar la estructura creativa e innovadora de planes de acción que permitan mejorar la calidad ambiental hacia el futuro.

\subsection{Costos ambientales y de seguridad}

La estimación de los costos asociados a la gestión de seguridad y medioambiente para la industria minera requiere de la arquitectura de una plataforma de diseño basada en la discriminación de costos entre el OPEX y el CAPEX de SMA a ser considerado. Esta consideración es 
esencial en la determinación de la asignación presupuestal del área funcional de SMA y es perfectamente compatible con la manera de evaluar la gestión en términos de costo por parte de la banca de inversión y los circuitos bursátiles nacionales e internacionales, así como en lo referente a las auditorías independientes de eficiencia en gestión ambiental y de seguridad minera. A continuación describiremos la estructura prevista para el OPEX y el CAPEX que determinan el costo anual de operación de una gestión de SMA en una industria minera convencional (véase la figura 5).

Figura 5

Estructura de costos

Costos de seguridad y medioambiente

Naturaleza

del costo

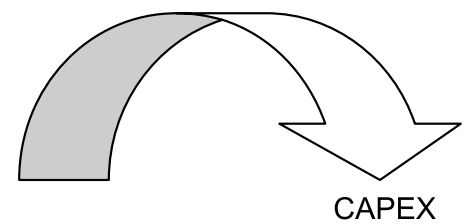

Costo total

Gestión de SMA
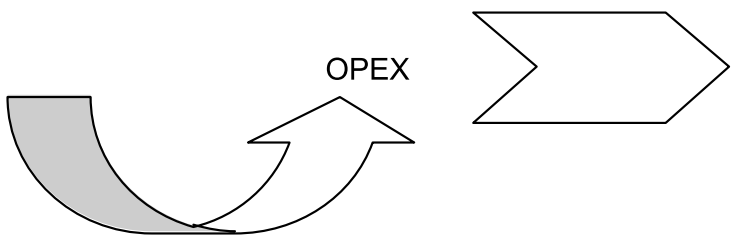

Componente de costo

(Componente general)

Planeamiento

Proyectos SMA

Gestión de proyectos

Cierre de mina

Reserva de riesgo

Control, monitoreo y medición Legal y regulatorio

Auditoría y revisión

Capacitación-entrenamiento

Elaboración propia.

El CAPEX de SMA está conformado por los costos derivados del planeamiento de proyectos y su ejecución como inversiones de la organización que permitirán obtener una mejor posición (posición de menor riesgo) frente al medioambiente y a la seguridad de los colaboradores de la organización. Las cuentas principales que componen el CAPEX son las siguientes: 
- Costos de planeamiento.

- Inversiones de proyectos ambientales y de seguridad.

- Costos asociados a la gestión de proyectos.

- Costos de cierre de mina.

- Reserva de riesgo para emergencias.

Los costos de planeamiento involucran la incorporación de variables ambientales y técnicas de seguridad con el fin de que los proyectos sean diseñados con un riesgo razonable para su operación; lo cual implica establecer condiciones para la población que puedan garantizar un mínimo impacto ambiental. Para ello se han determinado los siguientes componentes de costo:

- Costo de estudios preliminares.

- Costo de estudios de evaluación ambiental.

- Costo de análisis de riesgos de seguridad.

- Costo de estudios de ingeniería conceptual.

- Costo de estudios de prefactibilidad.

- Costo de estudios de ingeniería básica.

- Costo de estudios de factibilidad.

- Costo de estudios de ingeniería de detalle.

- Costo de estudios de impacto ambiental (EIA).

- Costo de programas de adecuación y manejo ambiental (PAMA).

- Costo de planes de cierre de mina.

- Costos derivados de otros estudios (hidrogeológicos, geotécnicos, etcétera).

Los costos correspondientes a inversiones en proyectos ambientales o de seguridad están referidos fundamentalmente a proyectos de sistemas de tratamiento de residuos, de mejora de procesos para optimizar recursos y de cierre progresivo (previos al cierre definitivo) mientras la mina se encuentra operando. En todos los casos se han determinado los siguientes componentes de costo:

- Costo de preparación, replanteo y movilizaciones.

- Costo de preparación y acondicionamiento de suelos.

- Costo de obras civiles para el proyecto.

- Costo de obras metalmecánicas. 
- Costo de obras eléctricas.

- Costo de obras de instrumentación y control.

- Costo de montaje, instalación, prueba y puesta en marcha.

Los costos asociados a la gestión de proyectos están enfocados a asegurar que los proyectos sean ejecutados conforme a las especificaciones del diseño y los fabricantes o modeladores de acuerdo con los estudios previos realizados. Los principales componentes de este costo son los siguientes:

- Costo de supervisión de obra.

- Costo de ensayos y pruebas requeridas para conformidad de supervisión.

- Costo de gerencia de administración de proyecto.

- Costo de consultoría especializada para revisar etapas del proyecto.

Los costos asociados al cierre de mina involucran los desembolsos previstos en el Plan de Cierre de Mina como costo de cierre definitivo. Es decir, la ejecución de los proyectos de cierre planeados y aprobados por el regulador que requieren ser específicamente aplicados para cada componente de cierre o instalación asociada. Estos costos incluyen:

- Costo de obras de remediación ambiental (geoquímica-física-social).

- Costo de sistemas activos de tratamiento para el cierre de mina.

- Costo de sistemas pasivos de tratamiento para el cierre de mina.

- Costo de restauración de suelos, revegetación, reforestación e impacto paisajístico.

- Costo de proyectos socioambientales de cierre de mina.

La reserva de riesgo para emergencias es una cuenta del CAPEX que debe ser prevista ante la probabilidad de ocurrencia de algún evento que pueda poner en condición de riesgo las operaciones actuales o que simplemente se considere en estado de emergencia ambiental o de seguridad de acuerdo a lo previsto en el análisis de riesgos. Ello incluye prevenir desastres naturales, inundaciones, desastres geotécnicos, embalses, cambio climático, negligencia y sabotaje, de ser el caso.

El OPEX de SMA está conformado por los costos de operación de seguridad y medioambiente necesarios para mantener el negocio en el día a día operativo. Las cuentas que conforman el OPEX son las siguientes (véase la figura 6): 


\section{Figura 6}

\section{Estructura de costos (CAPEX)}

Costo de estudios preliminares

Costo de estudios de evaluación ambiental

Costo de análisis de riesgos de seguridad

Costo de estudios de ingeniería conceptual

Planeamiento

Costo de estudios de prefactibilidad

(CV)

Costo de estudios de ingeniería básica

Costo de estudios de factibilidad

Costo de estudios de ingeniería de detalle

Costo de estudios de impacto ambiental (EIA)

Costo de PAMA

Costo de planes de cierre de mina

Costo otros estudios (hidrogeológicos, geotécnicos, etcétera)

Costo de preparación, replanteo y movilizaciones

Costo de preparación y acondicionamiento de suelos

Costo de obras civiles para el proyecto

Costo de obras metalmecánicas

Costo de obras eléctricas

Costo de obras de instrumentación y control

Costo de montaje, instalación, prueba y puesta en marcha

Costo de supervisión de obra

Gestión de

proyectos

(CV)

Reserva de riesgo

(CF)

Cierre de mina

(CF)
Costo de ensayos requeridos para conformidad

Costo de gerencia de administración de proyecto

Costo de consultoría especializada para revisión

Costo de obras de remediación ambiental

Costo de sistemas activos de tratamiento para el cierre

Costo de sistemas pasivos de tratamiento para el cierre

Costo de restauración de suelos, revegetación, reforestación

Costo de proyectos socioambientales de cierre de mina

Elaboración propia.

- Costos de control, monitoreo y medición.

- Costos regulatorios (fiscalizaciones, tasas, licencias, permisos y autorizaciones).

- Costos de auditoría/revisión.

- Costos de capacitación y entrenamiento.

Los costos asociados al control, monitoreo y medición involucran necesariamente las siguientes partidas de costo:

- Costo de muestreo.

- Costo de análisis de laboratorio y ensayos. 
- Costo de inspectores y logística de inspección.

- Costo de analistas de SMA.

- Costo de aseguramiento y control de calidad (QA/QC).

- Costo de calibración y mantenimiento de equipo de monitoreo.

Los costos regulatorios se derivan de las implicancias legales que incluyen el pago de aranceles, licencias y permisos, así como las tasas definidas por el Ministerio de Energía y Minas (MEM) bajo Fiscalización Minera de Seguridad y Medioambiente. Los principales componentes son los siguientes:

- Costo de licencia de uso de aguas.

- Costo de autorización de vertimientos.

- Costo de autorización de generación térmica.

- Tasa de fiscalización minera.

- Pagos de usufructos, convenios y regalías, etcétera.

- Costo de trámite para estudios ambientales.

- Costo de consultoría para la elaboración de informes regulatorios oficiales.

- Otros costos regulatorios formales asociados.

Los costos de auditoría y revisión son necesarios para asegurar una verificación sistemática y documentada de la gestión ambiental y de seguridad desempeñada en la organización. Los principales componentes de este costo son:

- Costo de revisión gerencial/revisión directiva.

- Costo de auditorías de sistemas de gestión (ISO14001, OHSAS18001, etcétera).

- Costo de auditorías técnicas especializadas (ambiental, geometalúrgica, geotécnica, geomecánica, hidráulica, hidrológica, hidrogeológica, sísmica, etcétera).

Los costos de capacitación y entrenamiento son esenciales para asegurar una correcta sensibilización y aseguramiento de la competencia profesional de los colaboradores vinculados a actividades críticas, sea por la naturaleza del peligro y riesgo involucrado o por su potencial impacto ambiental. La minería, por su naturaleza de riesgo, requiere de personal bastante calificado y competente, con un alto grado de sensibilización permanente en materia de riesgo para asegurar su control 
y gestión efectiva. Los principales componentes de este costo son generalmente los siguientes (véase la figura 7):

- Costo de programas de formación y capacitación de personal vinculado a elementos de riesgo críticos o aspectos ambientales significativos (AAS).

- Costo de programas de sensibilización en materia de seguridad o medioambiente a todos los colaboradores de la organización.

- Costo de cursos especiales para condiciones anormales y de emergencia.

- Otros cursos vinculados al área de seguridad y medioambiente.

\section{Figura 7}

Estructura de costos (OPEX)

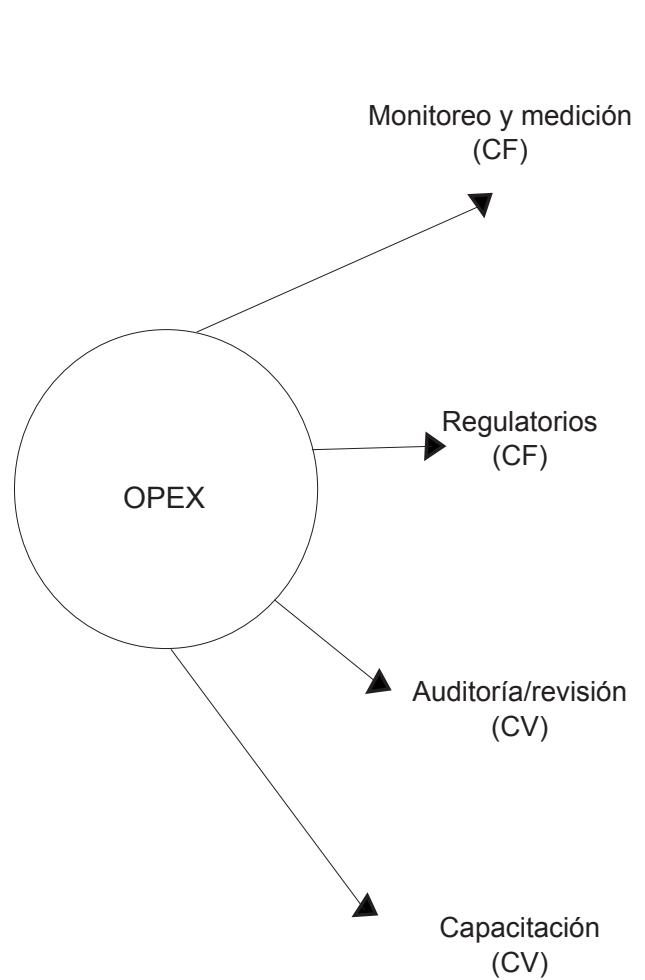

Costo de muestreo

Costo de análisis de laboratorio y ensayos

Costo de inspectores y logística de inspección

Costo de analistas de SMA

Costo de aseguramiento y control de calidad (QA/QC)

Costo de calibración y mantenimiento de equipo de monitoreo

Costo de licencia de uso de aguas

Costo de autorización de vertimientos

Costo de autorización de generación térmica

Tasa de fiscalización minera

Pagos de usufructos, convenios y regalías, etcétera

Costo de trámite para estudios ambientales

Costo de consultoría de informes regulatorios oficiales

Otros costos regulatorios formales asociados

Costo de revisión gerencial / revisión directiva

Costo de auditorías de sistemas de gestión

Costo de auditorías técnicas especializadas

Costo de programas de formación y capacitación

Costo de programas de sensibilización de SMA

Costo de cursos especiales / condiciones anormales / emergencia

Otros cursos vinculados al área de SMA

Elaboración propia. 


\section{POSIBLES APLICACIONES LATERALES}

Las aplicaciones laterales del presente desarrollo pueden ser perfectamente extendidas a cualquier tipo de organización que maneje recursos naturales agotables y que requiera una gestión de campo donde interactúen aspectos ambientales significativos como productos de sus actividades regulares.

De igual forma, este tipo de análisis puede ser perfectamente extendido a temas de gestión de responsabilidad social corporativa y manejo de relaciones comunitarias en los alrededores de la minería u otras actividades vinculadas al sector.

Finalmente, existen también aplicaciones contables y tributarias derivadas de la manera cómo contabilizar ingresos y costos ambientales que permiten deducir (de manera similar que la depreciación) los costos de agotamiento, convirtiéndose en un beneficio tributario real al momento del cálculo de los impuestos corrientes de la actividad minera.

\section{BIBLIOGRAFÍA}

British Standars Institution (2007). Norma Internacional de Sistemas de Gestión de Seguridad y Salud Ocupacional - OHSAS18001:2007. Londres: BSI.

British Standars Institution (2004). Norma Internacional de Sistemas de Gestión Ambiental ISO14001:2004. Londres: BSI.

British Standards Institution (1999). Norma Internacional Sistemas de Gestión de Seguridad y Salud Ocupacional -OHSAS18001:1999. Londres: BSI.

Business Information Group (2010). Canadian \& American Mines Handbook 2010-2011. Toronto.

Bustos Ayovi, Fernando (2010). Manual de gestión y control ambiental. 3. ${ }^{a}$ edición. Quito: R. N. Industria Gráfica.

Campodónico Sánchez, Humberto (1999). "Factores determinantes de la inversión real en América Latina: Las reformas estructurales en el sector minero peruano y las características de la inversión, 1992-2008”. Serie Reformas Económicas 24. Santiago de Chile: Cepal. 
Cascio, Joseph; Woodside, Gayle y Philip Mitchell (1997). Guía ISO14000. México D. F.: McGraw-Hill.

Folgar, Óscar (2005). Sistemas consolidados de gestión ISO9000, ISO14001 y OHSAS18001. Buenos Aires: Ediciones Macchi.

Friedman, Frank (2010). Practical guide to environmental management. 10. ${ }^{a}$ edición. Washington D.C.: Environmental Law Institute.

Hernández, Roberto; Fernández, Carlos y Pilar Baptista (2006). Metodología de la investigación. 4. ${ }^{\text {a }}$ edición. México D. F.: McGrawHill.

Ministerio de Energía y Minas del Perú (2010). Estadística minera. <www.minem.gob.pe>. [Consulta: 2 de noviembre del 2010.] 